\title{
Checklist clerking document improves health promotion among medical admissions
}

\author{
Sarah Cousins
}

\begin{abstract}
Health promotion, as defined by the Ottawa Charter (1) is the 'process of enabling people to increase control over, and to improve their health'. Four life style risk factors: smoking, alcohol, obesity and physical activity are recognised as leading to health inequalities. These factors have implications at an individual, community and national level and the burden of disease has large cost implications for the NHS and country as a whole. Therefore the assessment of these risk factors, and the provision of good health promotion assist to address these problems. A pilot and initial audit demonstrated that assessment for the key life style risk factors and the provision of health promotion was poor. This quality improvement audit developed an easy to use, checklist based, amended clerking document. This improved both assessment and provision of health promotion and will be carried forward to influence the designing of a new clerking document.
\end{abstract}

\section{Problem}

Many patients admitted to hospital have conditions which are preventable and caused by lifestyle factors. Health promotion is an important tool in reducing risk. On admission to hospital patients go through a clerking process, where information is gathered, including lifestyle risk factors. The data obtained is often incomplete, and health promotion is often not part of the history-taking process. It was felt that there was room for improvement of health promotion advice when people were admitted to hospital, through a brief intervention, and by setting up further input from specialists. Interventions, for example at a public health level, and the provision of health promotion at a local level, will hopefully reduce the prevalence of these lifestyle risk factors, and related conditions.

\section{Background}

Health promotion is defined by the Ottawa Charter (1) as 'the process of enabling people to increase control over, and to improve, their health.' Prevention, health and well-being, and health inequalities, are seen as key issues at a local and national level as championed by the NHS, WHO and NICE.

Engagement in health promotion aims to reduce human and economic costs associated with health inequalities, as laid out in the Marmot Review 'Fair Society, Healthy Lives.' (2) If everyone in England had the same death rates as the most advantaged, those currently dying prematurely as a result of health inequalities would, in total, have enjoyed between 1.3 and 2.5 million extra years of life. Additionally they would have had a further 2.8 million years free of limiting illness or disability. The additional NHS health costs are cited at in excess of $£ 3.3$ billion, as well as additional costs in welfare payments and lost tax revenue.

Many diseases that are encountered are influenced by lifestyle choices, particularly: smoking; alcohol consumption; diet and exercise. For example obesity has been shown to be directly responsible for over 9000 premature deaths a year in England. (2)

Interactions in hospitals are recognised as having the potential to cause behaviour change. For example there are large numbers of workers and service users, and therefore a large section of the population can be reached, and patients are amenable to education. (3) With the increase in chronic disease, health promotion is even more important in sustaining health, quality of life, and efficiency. However data from 2006-7 showed that only $4 \%$ of the NHS budget was spent on health prevention and promotion. (2)

NICE (4) have produced a number of documents aimed at encouraging health promotion via both its public health and clinical guidelines. Specifically there are NICE guidelines for obesity (PH42 2012, CG43 2006), smoking cessation (PH10 2008), alcohol dependence (PH24 2010, CG115 2011) and physical activity (PH8 2008).

\section{Baseline Measurement}

A pilot audit had been completed using 29 medical admissions to Sandwell Emergency Assessment Unit. This showed that $83 \%$ of patients had smoking status documented, $86 \%$ their alcohol status, $38 \%$ their body mass index (BMI) calculated, and $0 \%$ their physical activity level recorded. It went on to show that health promotion had been given to $14 \%$ of smokers, $11 \%$ of hazardous/harmful drinkers and $33 \%$ of obese/malnourished patients. $0 \%$ received advice regarding physical activity as no one had been assessed. Comparing these results to the 2012 National Health Promotion in Hospitals Audit standards (5) not one was met. It was felt that there was a significant problem with the assessment of lifestyle risk factors and provision of health promotion.

Therefore a larger audit was completed across the two hospitals that make up the Sandwell and West Birmingham NHS Trust. This Trust serves a population of 500,000 with 7500 staff. The clerking documents and nursing admission notes were reviewed for 50 
patients on each site who were admitted under the medical team to the adult assessment units. The results again showed that assessment and especially provision of health promotion was poor with $94 \%$ of patients having smoking status recorded, $86 \%$ alcohol status, $48 \% \mathrm{BMI}$ calculated and $0 \%$ physical activity. Health promotion was given to only $4 \%$ of smokers, $57 \%$ of hazardous/harmful drinkers and $15 \%$ of obese/malnourished patients. As physical activity was assessed in none of the patients, provision of advice regarding physical activity was again $0 \%$. In all cases this was only verbal advice.

See supplementary file: ds2180.pptx - "National Health Promotion in Hospitals Audit standards"

\section{Design}

It was felt that having the four key lifestyle factors on the clerking document, with a checklist approach and with a list of the available health promotion options displayed, would increase the recording and quality of information. This revised format would aid doctors completing the clerking document and remind them of the options available to provide health promotion. It was felt that this would be readily acceptable to the junior doctors as there was no additional paperwork, just a change of design. The intervention was felt to be intuitive, and time saving as a checklist approach would mean all the relevant information was to hand. The page was initially to be printed and stapled into the clerking document, with minimal cost implications and allowing for amendments prior to printing new clerking documents. As clerking documents are needed to complete assessment of patients it was felt to be sustainable, unlike temporary posters or formal education, which would need to be repeated with each new cohort of doctors.

\section{Strategy}

PDSA cycle 1: The results of the pilot audit were discussed at the Prevention Steering Group. The design of the proforma was discussed with the consultants on this committee and the junior doctor who undertook the pilot audit.

PDSA cycle 2: The results of the initial audit were presented, and the amended clerking document was discussed at the junior doctors meetings and journal club. Although they felt the new form would be beneficial the junior doctors were unclear as to the criteria levels for physical activity and for harmful and hazardous drinking. They felt that by including these on the form the completion rate would be greater.

PDSA Cycle 3: All junior doctors who clerked patients were informed of the new proforma. Subsequently, the proforma was implemented and a further audit carried out the following week.

See supplementary file: ds2182.docx - "PDSA Cycle"

\section{Post-Measurement}

After adjustment to the amended clerking document and education of junior doctors, a re-audit of another 100 patients was carried out. Once again this was 50 patients from each of the two hospitals within the Trust. Anecdotal evidence whilst collecting the data showed that the form was well accepted, with every form being at least partly completed, and none being torn out and the original used instead. Post implementation of the amended clerking document showed improvement in both the assessment of lifestyle risk factors and the provision of health promotion advice. Four of the eight standards were met, compared to two prior to the implementation. Additionally even those that did not meet the standards showed improvement. After the implementation of the amended document, smoking status was recorded in $98 \%$, and alcohol intake in $96 \%$. BMI was recorded in $33 \%$, and $44 \%$ of patients had physical activity recorded. Smoking cessation advice was provided to $61 \%$ of current smokers, and $81 \%$ of those drinking to a harmful or hazardous level were given health promotion advice. $0 \%$ of those with a raised BMI received information, and $20 \%$ of those who were physically inactive received advice. It was interesting to note that the type of health promotion had widened. Prior to the implementation of the amended document verbal advice had been the only type of health promotion given. With the amended document nicotine replacement was recorded as prescribed, information leaflets used and patients were referred to alcohol support services.

See supplementary file: ds2186.pptx - "Full results"

\section{Lessons and Limitations}

The numbers of notes reviewed in the different stages of the audit were large, meaning that the data should be robust. Two different hospital sites were used giving a larger variety of patients.

The amended clerking document includes areas which are currently still in development. Electronic referral routes are being developed and a health trainer is to be employed by the Trust. This meant that those completing the amended clerking document could have been confused by what health promotion referral options were available. However the junior doctors were provided with education surrounding the form and available referral options. Therefore the effect of this potential problem should be minimal. As the referral options develop continued education will be required.

Only medical notes were reviewed. Although medical admissions make up the greater proportion of those admitted to the assessment units there are also some surgical patients. These patients also need health promotion advice. Traditionally, surgical clerking documentation is more succinct, and therefore had we included surgical data in this audit the results are likely to have been adversely affected. The same clerking document is used by both specialties, and many junior doctors rotate through medicine and surgery, therefore the benefit of the amended form should pass onto to those admitted under surgery.

There is the possibility of selection bias for recording data on the assessment units, which may be related to additional events to raise awareness prior to the launch of the checklist. Nevertheless, the differences in rates of assessment for physical activity and 


\section{BMJ Quality Improvement Reports}

intervention for smoking were large, suggesting a clinically relevant benefit.

The results for assessment of $\mathrm{BMI}$ and for the provision of health promotion decreased between the initial audit and the re-audit. In the initial audit the percentage having BMl assessed was quite high (it was one of only two areas that met the standard), and perhaps this was an anomalous result, it was noted that the assessment of weight was done much better at one of the hospitals within the Trust than the other in the re-audit, and so the average result was pulled down. Those patients that needed weight advice was also much lower in the re-audit (12 patients compared to 27 ).

As found in the progress flow the same data can be collected by four different groups and is currently recorded in up to 3 different documents. The Trust is currently in the process of redesigning the clerking document. This will be one document which is started in A\&E and is then used by nursing staff and the medical team. As further information is gathered it is then written in the same form, rather than it being duplicated. This has both cost and efficiency implications. The information gained from this project is being used in the development of this new document.

\section{Conclusion}

This project was started as it was found that documentation of assessment of lifestyle risk factors and then provision of health promotion advice was poor, with the use of the existing clerking document. Given the importance of health promotion and its benefits to individuals, communities and the country as a whole there was a clear need to improve the health promotion provision within the Trust. This project has shown that redesigning the clerking document can have beneficial effects in assessing lifestyle risk factors including smoking, alcohol and physical activity, and that health promotion can be improved with an easy and cheap intervention.

\section{References}

1 WHO. The Ottawa Charter for Health Promotion. World Health Organisation. 1986 [Cited 2013 August 11] Available from: http://www.who.int/healthpromotion/

2 Marmot M. Fair Society, Health Lives, The Marmot Review, Strategic Review of Health Inequalities in England. 2010. [Cited 2013 August 11]. Available from: http://www.marmotreview.org.

$3 \mathrm{WHO}$ /Europe. The International Network of Health Promoting Hospitals and Health Services: Integrating health promotion into hospitals. 2007 [Cited 2013 August 11]. Available from: http://www.euro.who.int

4 National Institute for Health and Care Excellence. [Cited 2013 August 11] Available from: http://www.nice.org.uk

5 National Health Promotion in Hospitals Audit, Acute and Specialist Trusts 2012 [Cited 2013 August 11] Available from: http://www.nhphaudit.org

\section{Declaration of interests}

Nothing to declare

\section{Acknowledgements}

Dr Douglas Robertson, Dr Kathryn Basnayake, Dr Rory Gillingham 